\title{
Preliminary Dynamic Feasibility and Analysis of a Spherical, Wind-Driven (Tumbleweed), Martian Rover
}

\author{
John J. Flick* \\ NASA Langley Research Center, Hampton, Virginia, 23681 \\ Matthew D. Toniolo ${ }^{\dagger}$ \\ Analytical Mechanics Associates Inc., Hampton, Virginia, 23666
}

\begin{abstract}
The process and findings are presented from a preliminary feasibility study examining the dynamics characteristics of a spherical wind-driven (or Tumbleweed) rover, which is intended for exploration of the Martian surface. The results of an initial feasibility study involving several worst-case mobility situations that a Tumbleweed rover might encounter on the surface of Mars are discussed. Additional topics include the evaluation of several commercially available analysis software packages that were examined as possible platforms for the development of a Monte Carlo Tumbleweed mission simulation tool. This evaluation lead to the development of the Mars Tumbleweed Monte Carlo Simulator (or Tumbleweed Simulator) using the Vortex physics software package from CM-Labs, Inc. Discussions regarding the development and evaluation of the Tumbleweed Simulator, as well as the results of a preliminary analysis using the tool are also presented. Finally, a brief conclusions section is presented.
\end{abstract}

\section{Introduction}

$\mathrm{T}$ HIS document is a presentation of the findings from a feasibility study and preliminary dynamic analysis of a wind-driven rover concept for the exploration of the surface of Mars. This wind-driven rover concept was given the name Mars Tumbleweed. The feasibility studies were performed on a very fundamental level and were intended to help form a basic understanding of how a Tumbleweed rover might behave in several static and dynamic situations. The results of these feasibility studies led to the evaluation of several commercial off the shelf (COTS) analysis tools and the development of a dynamic simulation tool for the Tumbleweed concept using one of these COTS programs, the Vortex physics software package from CM-Labs, Inc. This tool has been named the Mars Tumbleweed Monte Carlo Simulator or Tumbleweed Simulator for short. This paper concludes with a discussion of results obtained from an evaluation of the Tumbleweed Simulator and a preliminary analysis using the tool.

The feasibility studies and the development of the Tumbleweed Simulator have helped to build a fundamental understanding of the Tumbleweed dynamics problem and to guide future analysis and tool development efforts. Additionally, these activities have helped to indicate, from a basic physics standpoint, that the Tumbleweed rover concept is a feasible means for exploring vast regions of the Martian surface and an innovative conceptual exploration vehicle and payload platform for the Martian surface.

\section{Preliminary Feasibility Studies}

The feasibility studies discussed in this paper build on the results of several initial feasibility studies performed by the Langley Research Center (LaRC) Tumbleweed team in FY $2002^{1}$. These studies continue to explore the necessary Tumbleweed rover properties for impending motion in a variety of worse-case situations. The feasibility studies discussed in this paper focus on two specific situations: static analysis of a Tumbleweed rover trapped on a rocky slope and a simplified dynamics analysis of a Tumbleweed rover impacting a rock. The following sections discuss these two scenarios in more detail.

\footnotetext{
${ }^{*}$ Aerospace Engineer, Architectures Missions \& Science Branch (SACD), MS 462, AIAA Professional Member.

${ }^{\dagger}$ Project Engineer, AMA Inc., 303 Butler Farm Rd. Suite 104A, Non-Member.
} 


\section{A. Static Analysis of a Tumbleweed Rover Trapped on a Rocky Slope}

On the Martian surface, wind speeds and directions are constantly varying. Velocities vary from $2 \mathrm{~m} / \mathrm{s}$ to greater than $25 \mathrm{~m} / \mathrm{s}$ depending on the season, time of day, and weather conditions ${ }^{1}$. Winds typically cease all together during night hours. Consider a Tumbleweed rover traveling along the Martian surface being pushed by a $7 \mathrm{~m} / \mathrm{s}$ afternoon breeze. The Sun will eventually set, the winds will die down, and the rover will probably stop moving until the winds pick up again the next day. As a worst-case scenario, the Tumbleweed could possibly stop on a slope and become lodged between several rocks. What wind speed will then be required to nudge that Tumbleweed up out of its resting place? Is a wind of this speed one the Tumbleweed is likely to encounter? What slopes and rock sizes can a particular Tumbleweed rover overcome? Will the Tumbleweed rover be able to dislodge itself and continue its mission up the slope after becoming trapped as described above? This feasibility study attempted to answer those questions.

Figure 1 shows a free body diagram of a Tumbleweed rover trapped on a slope of angle. The Tumbleweed rover is assumed to be a flexible sphere. As the wind force exerted on the Tumbleweed increases, the weight that was distributed at rest through points $\mathrm{G}, \mathrm{P}$, and the small down slope rock begins to shift over to point $\mathrm{P}$ exclusively on the up slope rock face. This continues through increasing wind velocities until finally the Tumbleweed's entire weight is distributed onto the surface of the up slope rock, approximated as acting point $\mathrm{P}$ for this analysis. At this stage of the transition, any increase in wind velocity will enable the Tumbleweed to overcome, or roll over top of, the up slope rock and continue. Therefore, when summing moments about point $\mathrm{P}$, Eq. (1) defines when the wind force acting on the Tumbleweed is sufficient to push the rover past the up slope rock.

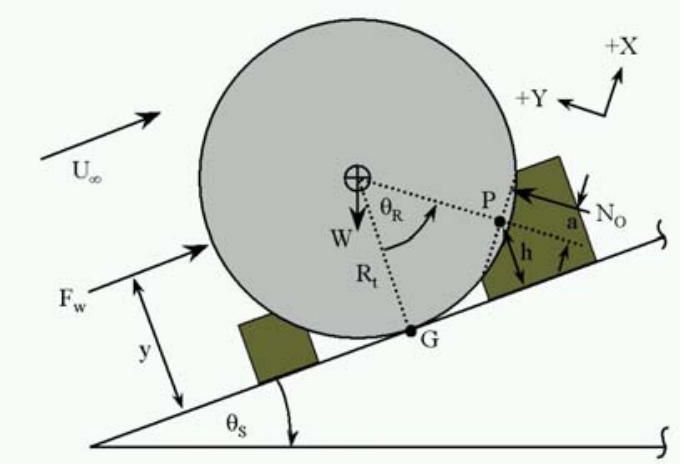

Figure 1. Free body diagram of Tumbleweed rover trapped on a sloped surface.

$$
M_{F_{W}}>M_{W}+M_{N_{O}}
$$

$M_{F w}$ is the moment about point $\mathrm{P}$ exerted on the Tumbleweed as a result of the wind force $\left(F_{w}\right)$ and is defined by Eq. (2). $M_{W}$ is the moment about point $\mathrm{P}$ caused by the force of gravity pulling the Tumbleweed down slope and is defined by Eq. (3). $M_{N o}$ is the moment about point $\mathrm{P}$ caused by the flexible Tumbleweed's rolling resistance and is defined by Eq. (4). Rolling resistance here is approximated as a moment opposing the Tumbleweed's forward motion generated by an offset normal force $\left(N_{o}\right)$ some distance from point $\mathrm{P}$ at the center of the contact patch $(a)$ and is related to the coefficient of rolling resistance $\left(\mu_{r}\right)$ by Eq. (5). More information about this relationship can be found in Ref. 2.

$$
\begin{gathered}
M_{F_{W}}=\left[C_{D} A_{r e f} \frac{1}{2} \rho U_{\infty}^{2} \sin \left(90-\theta_{R}\right)\right]\left(R_{t}-c\right) \\
M_{W}=W_{X}(R t-c) \\
M_{N_{O}}=N_{O} a \\
a=\mu_{r} R_{t} \\
N_{O}=\left[W \cos \left(\theta_{R}+\theta_{S}\right)\right]+\left[C_{D} A_{r e f} \frac{1}{2} \rho U_{\infty}^{2} \cos \left(\theta_{R}+\theta_{S}\right)\right]
\end{gathered}
$$




$$
\begin{gathered}
c=2 \mu_{r}^{2} R_{t} \\
W_{X}=W \sin \left(\theta_{R}+\theta_{S}\right)
\end{gathered}
$$

The variables for Eqs. 2 through 8 that have not been previously mentioned are defined as follows: $C_{D}$ is the drag coefficient of a given Tumbleweed vehicle, $A_{\text {ref }}$ is its cross-sectional area, $\rho$ is the atmospheric density on Mars, $U_{\infty}$ is the free-stream velocity of the wind, $\theta_{R}$ is the rock angle used to define the size of the up slope rock, $R_{t}$ is the radius of the Tumbleweed, $c$ is the deformation distance of the rover's structure under loading, $W$ is the weight of the Tumbleweed under Martian conditions, $\theta_{S}$ is the slope angle, and $W_{X}$ is the weight component of the rover in the $x$ direction.

For typical aircraft tires, ranges for $\mu_{r}$ can be anywhere from 0.02 to 0.3 depending on the flexibility of the tire and the rolling surface conditions ${ }^{2}$. The range considered in this study was assumed to be 0 to 0.15 . Validation of this assumption is discussed in Ref. 3. It is assumed for this analysis that the wind force acts through the Tumbleweed's center of gravity. In reality, the center of pressure would be offset somewhat due to boundary layer effects. However, presently the significance of these boundary layer effects is unclear.

Figures 2 and 3 show wind speed versus rock height for a particular Tumbleweed rover configuration on flat ground and on a $30^{\circ}$ slope, respectively. The Tumbleweed's radius was varied from $1 \mathrm{~m}$ to $4 \mathrm{~m}$, while the mass, drag coefficient, and coefficient of rolling resistance were held constant. However, any of the Tumbleweed design parameters described above can be varied within the analysis codes developed for this study. The drag coefficient of 1 was selected based on values obtained from the wind tunnel testing performed on a variety of Tumbleweed designs at $\mathrm{LaRC}^{4}$. The mass $\left(M_{t}\right)$ was arbitrarily chosen. The coefficient of rolling resistances was selected to be the worst-case value expected for the range selected earlier.

Consider the data for the $3 \mathrm{~m}$ radius Tumbleweed displayed in Fig. 2. On flat ground, a $16 \mathrm{~m} / \mathrm{s}$ wind will be required to overcome a $1 \mathrm{~m}$ high rock. As expected, for a $4 \mathrm{~m}$ radius Tumbleweed, a slower wind, roughly $10 \mathrm{~m} / \mathrm{s}$, will be needed to overcome the same $1 \mathrm{~m}$ rock. We also see that the $1 \mathrm{~m}$ and $2 \mathrm{~m}$ radius Tumbleweeds are, at best, only capable of overcoming relatively small rocks at very high wind speeds.

Now consider the same $3 \mathrm{~m}$ Tumbleweed in Fig. 3 where there is now a $30^{\circ}$ slope to overcome, as well as various rocks. For lower wind speeds, the $3 \mathrm{~m}$ Tumbleweed now requires a $17 \mathrm{~m} / \mathrm{s}$ wind to overcome that same $1 \mathrm{~m}$ high rock. However, by comparing these two charts, one notices the wind speed requirements for conquering a rock on flat ground and conquering a rock on a sloped surface become similar as the velocity increases. In other words, the $4 \mathrm{~m}$ Tumbleweed, regardless of whether or not it is on a flat or sloped surface, requires a $20 \mathrm{~m} / \mathrm{s}$ wind to overcome a $2.5 \mathrm{~m}$ high rock. The same trend is apparent for the $3 \mathrm{~m}$ Tumbleweed when other design parameters such as mass and drag coefficient are varied. This implies that for slopes $\leq 30^{\circ}$, as the wind speed approaches $20 \mathrm{~m} / \mathrm{s}$, the moment created by the wind force $\left(M_{F w}\right)$ becomes dominant, eventually negating the effects of gravity pulling the Tumbleweed back down the slope. For slopes $>30^{\circ}$, this trend would eventually appear as well, but at higher wind speeds.

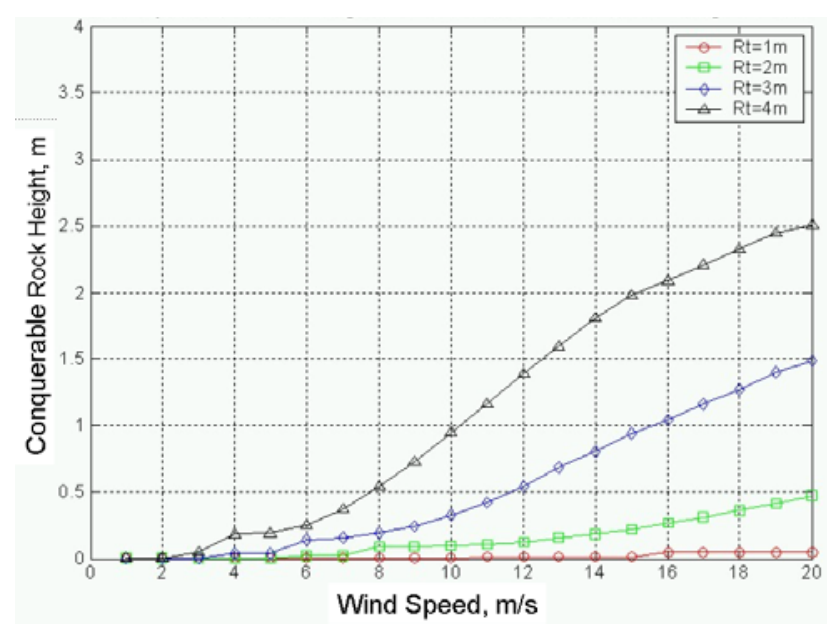

Figure 2. Wind speed vs. rock height for a Tumbleweed on flat ground; $M_{t}=10 \mathrm{~kg}, C_{D}=1, \mu_{r}$ $=0.15$.

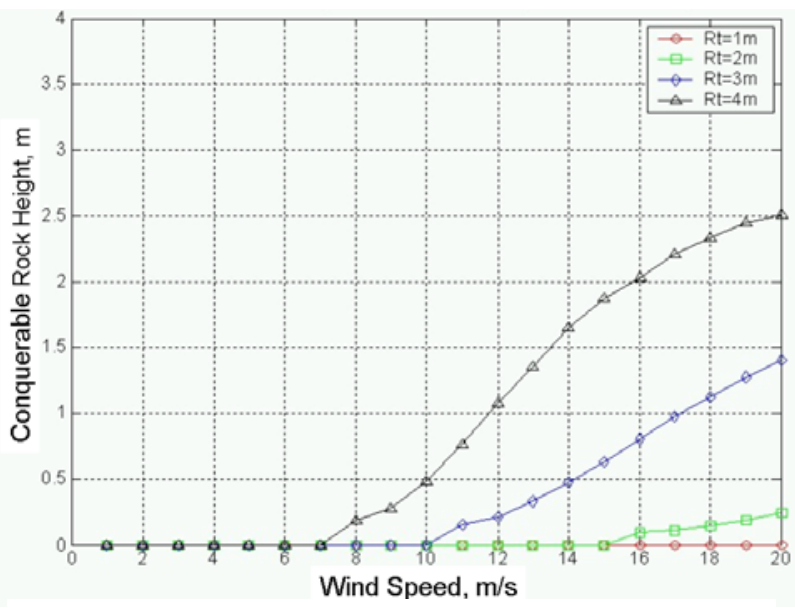

Figure 3. Wind speed vs. rock height for a Tumbleweed on a $30^{\circ}$ slope; $M_{t}=10 \mathrm{~kg}, C_{D}=1, \mu_{r}$ $=0.15$. 
This feasibility study shows that Tumbleweed rovers of reasonable size and mass can overcome substantial rock impedances on relatively extreme slopes. A $30^{\circ}$ slope on Mars is not common over long distances and is a conservative estimate of a worst-case condition. Similarly, the large rocks considered in this study ( $1 \mathrm{~m}$ to $2.5 \mathrm{~m})$ are not common features on the Martian surface and would also be conservative, worst-case condition assumptions. The wind speeds required to dislodge a Tumbleweed rover in this situation (approximately $10 \mathrm{~m} / \mathrm{s}$ to $20 \mathrm{~m} / \mathrm{s}$ depending on the slope angle, rock size, and Tumbleweed configuration) are also not common. However, they are not unheard of. Wind speeds average from $2 \mathrm{~m} / \mathrm{s}$ to $5 \mathrm{~m} / \mathrm{s}$ during the day with relatively strong gusts of $10 \mathrm{~m} / \mathrm{s}$ to $20 \mathrm{~m} / \mathrm{s}$. Seasonal dust storms can produce winds exceeding $25 \mathrm{~m} / \mathrm{s}^{1}$. Therefore, a lodged Tumbleweed rover may have to wait for a sufficiently strong wind to push it out of its entrapment, but across most areas of Mars it would be difficult to render itself permanently immobile.

\section{B. Simplified Dynamic Analysis of a Tumbleweed Rover Impacting a Rock}

An additional feasibility study was undertaken to examine, on a preliminary level, the fundamental dynamics of a Tumbleweed rover impacting a rock. Consider a Tumbleweed rover rolling on a flat surface at a given velocity and exposed to a constant wind. Somewhere along the way the Tumbleweed impacts a rock and bounces up into the free stream. What is the significance of the wind pushing the Tumbleweed downrange compared to the same scenario in a zero wind environment? What pre-impact velocity is required so that the rover will bounce up and over the rock and not backward in the opposite direction from which it came from? This feasibility study attempts to answer these questions.

Figure 4 shows the free body diagram of a Tumbleweed rover impacting a rock. The Tumbleweed and the rock are assumed to be rigid structures. Collisions between the Tumbleweed and the rock and the Tumbleweed and the ground were modeled as "billiard ball" collisions with assumed coefficients of restitution. Before impact with the rock, the Tumbleweed has some initial velocity $\left(V_{i}\right)$. After impact with the rock, the Tumbleweed will have some final velocity $\left(V_{f}\right)$ in the direction $\theta_{T}$ from the horizontal. The rock is fixed to the ground and does not acquire any velocity after the collision. The initial velocity vector can be broken up into components that are tangential ( $t$ direction) and normal ( $n$ direction) to the point of impact (P). It then follows from simple collision physics that the post and pre impact velocities as well as the magnitude and direction of the post impact velocity vector are related by Eqs. 9 to 12 .

$$
\begin{gathered}
V_{f_{t}}=V_{i_{t}} \\
V_{f_{n}}=-e V_{i_{n}} \\
V_{f}=\sqrt{V_{f_{t}}^{2}+V_{f_{n}}^{2}} \\
\theta_{T}=\theta_{R}+\tan ^{-1}\left(V_{f_{n}} / V_{f_{t}}\right)
\end{gathered}
$$

$V_{f t}$ and $V_{i t}$ are the post and pre impact velocities of the Tumbleweed in the $t$ direction, respectively. $V_{f n}$ and $V_{i n}$ are the post and pre impact velocities of the Tumbleweed in the $n$ direction. The coefficient of restitution is defined by $e$ and is arbitrarily assumed to be 0.7 for both rock and ground Tumbleweed impacts. $V_{f}$ is the magnitude of the post impact velocity vector, and $\theta_{T}$ is the post impact trajectory angle. It is necessary to note here that assumptions regarding structural properties of the Tumbleweed, such as flexibility and coefficient of restitution, are arbitrary at this point in the project. Further development of the rover's structural design parameters, such as mass, size, and 
construction materials, will be required to move to higher fidelity dynamics analyses. However, the assumptions made were deemed adequate for this preliminary feasibility study.

From the final velocity and trajectory angle, it is then possible to compute the Tumbleweed's post impact, normal projectile trajectory. Going one step further, the contribution of the free stream wind to the Tumbleweed's down range motion can also be approximated by accounting for the relative wind velocity acting on the Tumbleweed. Figure 5 shows the trajectory of a particular Tumbleweed configuration impacting a rock defined by a $30^{\circ}$ rock angle, which translates to a rock of approximately $0.4 \mathrm{~m}$ in height. The solid line shows the post impact

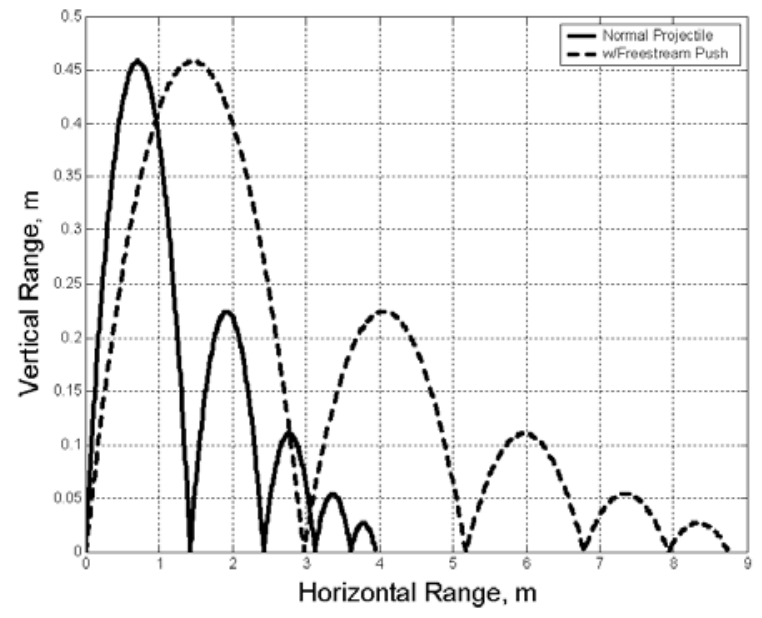

Figure 5. Tumbleweed trajectory; $R_{t}=3 \mathrm{~m}, M_{t}=10 \mathrm{~kg}$, $C_{D}=0.5, e=0.7, \theta_{R}=30^{\circ}, V_{i}=2.5 \mathrm{~m} / \mathrm{s}, U_{\infty}=6 \mathrm{~m} / \mathrm{s}$ trajectory of the Tumbleweed if there were no wind contributing to down range motion (the motion of a normal projectile). The dashed line shows the post impact trajectory of the Tumbleweed accounting for the down range contribution of the free stream wind push. In a $6 \mathrm{~m} / \mathrm{s}$ wind, the wind contribution is significant, doubling the achieved down range motion in the same amount of bounces.

Figure 5 also shows that the initial velocity of the Tumbleweed was sufficient enough to allow the Tumbleweed to bounce high enough, approximately $0.45 \mathrm{~m}$, to clear the top of the $0.4 \mathrm{~m}$ rock. Additionally, it can be seen that the trajectory angle, $\theta_{T}$, is less than $90^{\circ}$. This maintains the forward momentum of the Tumbleweed enabling it to progress over the rock instead of bounce off in the opposite direction from which it originally came. From this simple analysis, rough approximations can be made for initial Tumbleweed velocity requirements necessary to dynamically conquer rocks of various sizes.

\section{Commercial Off the Shelf (COTS) Analysis Tool Study}

Several COTS software packages were studied in order to evaluate their dynamics analysis capabilities and potential application to the Tumbleweed dynamics problem. More specifically, the main objective of this aspect of the preliminary dynamics study was to evaluate the fidelity of each COTS package when used for modeling sphereto-surface interactions to simulate a Mars Tumbleweed rover. If a capable COTS software package were found, it would be a valuable contribution to the ongoing development of an in-house Tumbleweed dynamics analysis tool, as well as to the dynamics analysis activity as a whole, by serving as an additional independent modeling tool. The three software packages that became the focus of the study were Maya (Alias/Wavefront), ADAMS (MSC Software), and Vortex (CM-Labs).

\section{A. Testing of the COTS Analysis Tools}

Due to the nonlinear nature and complexity of a full-scale simulation of a Tumbleweed rover in motion, it is difficult to determine the accuracy of any analysis output directly. Instead, several test cases that have well known and easy to determine analytical solutions were designed. These test cases were then assembled within each software package and their output results compared to the known analytical solutions. While passing each of these tests does not ensure fidelity in the full-scale simulation, failing one or more of the tests clearly indicates weaknesses in the tool's analysis abilities. A basic description and the assumptions for each test follow.

\section{Translation}

The translational motion of an object must obey Eq. (13), where $F$ is the force acting on the Tumbleweed, $m$ is the mass, and $a$ is the acceleration.

$$
F=m a
$$




\section{Rotation}

The rotational motion of an object must obey Eq. (14), where $T$ is the torque acting on the Tumbleweed, $I$ is the mass moment of inertia, and $\alpha$ is the angular acceleration.

$$
T=I \alpha
$$

\section{Friction}

Within the analysis tool, it must be possible to model static and dynamic friction. Static friction must allow specification of a constant force that must be overcome before sliding motion starts. Dynamic friction must obey Eq. (15).

$$
F_{F}=\eta N
$$

$F_{F}$ is the friction force, $\eta$ is the coefficient of friction, and $N$ is the normal force. For a rolling body, it must be possible to specify both an initial rolling resistance, which must be overcome before the body will move, and a dynamic rolling resistance separate from the dynamic friction which opposes the sliding of the rolling body.

It is necessary to note here that dynamic friction is not the same as rolling resistance. Dynamic friction is the result of one surface moving over another and this does not occur for a body rolling without slipping. Rolling resistance arises due to other effects, mainly flexibility of the rolling object, which leads to deformations and energy dissipation. This is the same as the rolling resistance discussed earlier for the first feasibility study in section II-A. Static friction does not prevent a rolling body from moving; it causes it to roll without slipping.

\section{Gravity}

Within the analysis tool, it must be possible to model the effects of gravity and be able to specify the field strength to that of Mars.

\section{Wind}

It must be possible to model a constant wind force and to be able to introduce random gusts. The wind force of an object must obey Eq. (16).

$$
F_{W}=C_{D} A_{r e f} \frac{1}{2} \rho U_{r}^{2}
$$

$U_{r}$ is the relative speed between the wind and Tumbleweed rover and all other variables are as previously defined.

\section{Collisions}

Within the analysis tool, it must be possible to model the collision of a moving object with a static object of arbitrary shape. It must also be possible to specify the coefficient of restitution between two impacting surfaces.

\section{Terrain}

Within the analysis tool, it must be possible to import a representation of terrain via a terrain database and model collisions of a moving object with the imported terrain.

\section{B. Summary of Activity and Results}

Table 1 summarizes the results of the COTS analysis tool study discussed in this section. Each entry labeled as Pass indicates that the test results agreed with the known analytical solutions, and that little or no special measures were needed to make the software in question work properly. Entries labeled as Fail indicate that the software was incapable of reproducing the known analytical solutions or that it was impractical to model the problem correctly within the tool for that particular test case. Entries labeled Marginal indicate that the software only worked on some levels for a particular test case.

As a result of this study, the Vortex software package was identified as the best choice for analysis of the Tumbleweed rover dynamics problem. A developmental analysis tool was developed around the Vortex software package and is discussed further in section IV of this paper. It should be noted that since the development of the Vortex application, several prior issues with the ADAMS software have since been resolved. For various reasons 
the ADAMS software could provide a higher fidelity analysis capability. An analysis tool using the ADAMS software package could possibly be developed in the future activities.

Table 1. Summary of COTS Analysis Tool Study Results

\begin{tabular}{|l|l|l|l|l|l|l|l|l|}
\hline & Translation & Rotation & Friction & Gravity & Wind & Collisions & Terrain & Slide-to-Roll \\
\hline Maya & Pass & Fail & Pass & Pass & Fail & Marginal & Pass & N/A \\
\hline ADAMS & Pass & Pass & Pass & Pass & Pass & Fail & Marginal & Pass \\
\hline Vortex & Pass & Pass & Marginal & Pass & Pass & Pass & Pass & Pass \\
\hline
\end{tabular}

\section{Summary of COTS Tool Shortcomings}

MAYA Shortcomings:

1) Rotation Test: Fail

Moments of inertia cannot be specified. Furthermore, the default values for the moments of inertia of objects are inaccurate. For a uniform density sphere and a thin walled hollow sphere the MAYA default moments of inertia are off by factors of 3.36 and 2 , respectively.

2) Wind Test: Fail

The net force acting on the object is treated as being linearly proportional to the relative velocity of the object. This is incorrect. The net force of the wind acting on an object should be proportional to the square of the relative velocity.

3) Collisions Test: Marginal

Coefficient of restitution not properly defined.

ADAMS Shortcomings:

1) Collisions Test: Fail

Proper trajectory of a bouncing ball with a specified coefficient of restitution could not be reproduced.

2) Terrain Test: Marginal

Without the ADAMS/Exchange module, the ADAMS/Solver will only accept CAD files of a Parasolid format (from Unigraphics). In addition, ADAMS exhibited some instability regarding certain Parasolid files.

Vortex Shortcomings:

1) Friction Test: Marginal

Vortex only uses a single coefficient of friction and does not model static friction separately from dynamic friction.

\section{Evaluation of the Mars Tumbleweed Monte Carlo Simulator}

Using Visual C++ 6.0, a wrapper application called the Mars Tumbleweed Monte Carlo Simulator (Tumbleweed Simulator), was created to drive the Vortex simulation software. The current developmental version of the Tumbleweed Simulator allows the user to perform preliminary Monte Carlo simulations for the purpose of evaluating the statistical distributions of Tumbleweed rovers over a Mars relevant terrain. In other words, a virtual environment can be created within the tool where the performance of various Tumbleweed rover design configurations can be examined.

A comprehensive evaluation of the developmental Tumbleweed Simulator was performed to determine the current analysis capabilities of the tool and to identify areas for future improvement. Several different Tumbleweed rover design configurations and a Mars relevant terrain were modeled within the Tumbleweed Simulator. The evaluation was structured to answer three basic questions. Each of these questions is addressed in the sections below. Each section is concluded with a description of the identified improvement areas for the Tumbleweed Simulator that are relevant to that particular question.

\section{Question 1: Can a realistic Mars environment be modeled within the simulator?}

The Tumbleweed Simulator allows the user to define terrain size, rectangular rock dimensions for an upper bound large rock and a lower bound small rock, the number of rocks at each boundary that will be included on the terrain, and a number of rectangular rocks sized randomly in between the specified upper and lower bounds. The current version of the simulator includes one other terrain option that allows the user to enter in a specified terrain profile, which in general would consist of a two dimensional array of vertical heights. This option could be used to create riverbeds, craters, or other sloping surfaces. However, simulating a Mars relevant rock field is the most 


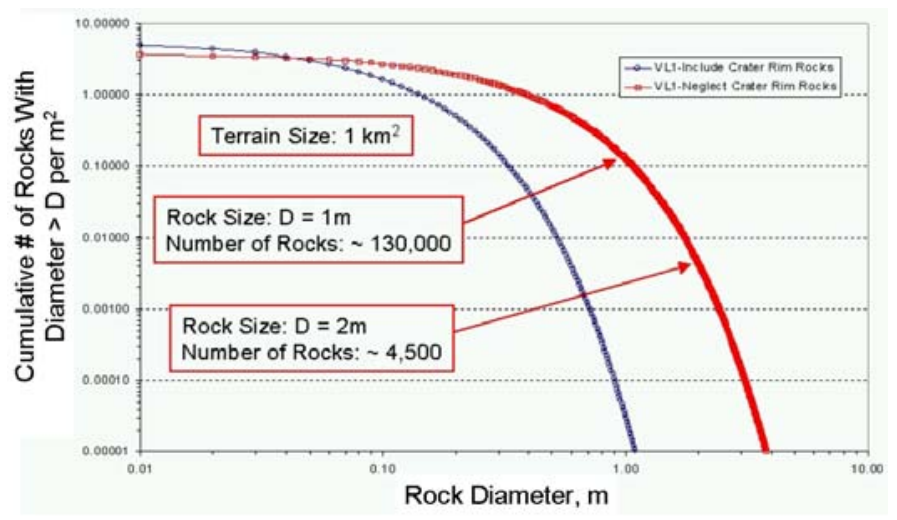

Figure 6. Cumulative number of rocks vs. rock diameter for the Viking 1 landing site ${ }^{5}$. difficult terrain-modeling challenge, as well as the toughest to analyze. Therefore, for this evaluation, the capability of the Tumbleweed Simulator to model a rocky Martian plain was the focus.

At the time of this analysis, detailed, highresolution terrain data for Mars was limited to the landing locations of the Viking and Pathfinder missions. For this evaluation, the Viking 1 landing site was chosen. Figure 6 shows the cumulative number of rocks per square meter as a function of rock diameter. The curves on this figure were plotted from mathematical expressions derived from stereo measurements at the Viking 1 landing site ${ }^{5}$.

As discussed above, the current version of the Tumbleweed Simulator only provides the user with control over the number and dimensions of the upper and lower bound rocks. If the user then wishes to place rocks of different dimensions onto the terrain their only option is to specify a number of rocks to be randomly sized somewhere in between the dimensions of the already specified upper and lower bound rocks. Working within this restriction, a number of large rocks $(2 \mathrm{~m}$ cubes) and small rocks ( $1 \mathrm{~m}$ cubes) were chosen for the terrain. These sizes were chosen in order to create a rock field that would be difficult for the Tumbleweed rover to navigate. The idea was that the difficult terrain would challenge the capabilities of the Tumbleweed Simulator while at the same time offer an interesting environment to observe the dynamics of the Tumbleweed concept.

From Fig. 6, it can be seen that on a $1 \mathrm{~km}^{2}$ terrain there should be approximately 130,000 rocks of $1 \mathrm{~m}$ diameter and 4,500 rocks of $2 \mathrm{~m}$ diameter. The distribution neglecting the crater rim rocks was selected because it allows for higher numbers of large rocks and was better suited for the difficult terrain desired. Computer memory restrictions only allowed a total of 10,000 rocks to be included on the $1 \mathrm{~km}^{2}$ terrain. This memory problem is a fundamental issue with the way the Vortex software is set up and will not allow the modeling of large terrains with large numbers of rocks. As a result, the terrain was restricted to 4,500 rocks of $2 \mathrm{~m}$ diameter and 5,500 rocks of $1 \mathrm{~m}$ diameter. Figures 7 and 8 show a photograph of the actual Viking 1 landing site and the virtual terrain generated within the Tumbleweed Simulator, respectively.

Winds were modeled at a constant velocity and direction. The velocity chosen was an average Martian velocity of $7 \mathrm{~m} / \mathrm{s}$. The current version of the Tumbleweed Simulator does not account for boundary layer effects or allow the user to define winds of constantly varying magnitude and direction.

Several improvement areas were identified regarding the environmental modeling capabilities of the Tumbleweed Simulator. The memory problem present in the current version of the tool when generating large terrains with large numbers of rocks is a major restriction to modeling a realistic Mars terrain. The idea of the Tumbleweed concept is to traverse vast and possibly highly rugged areas of the Martian surface. Any mission could potentially cover hundreds if not thousands of kilometers. Alternative methods for terrain modeling within the tool will have to be explored. For example, one possible alternative may be to only generate small patches of the terrain as a Tumbleweed rover travels through it. This option would free up memory resources since only small terrain areas

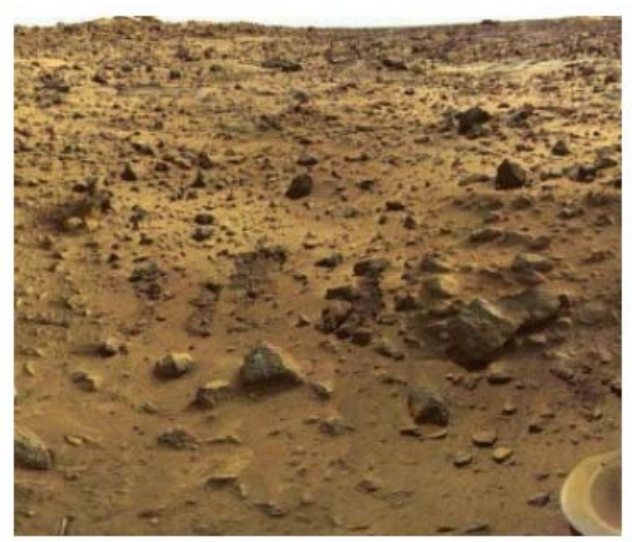

Figure 7. Photograph of the Viking 1 landing site.

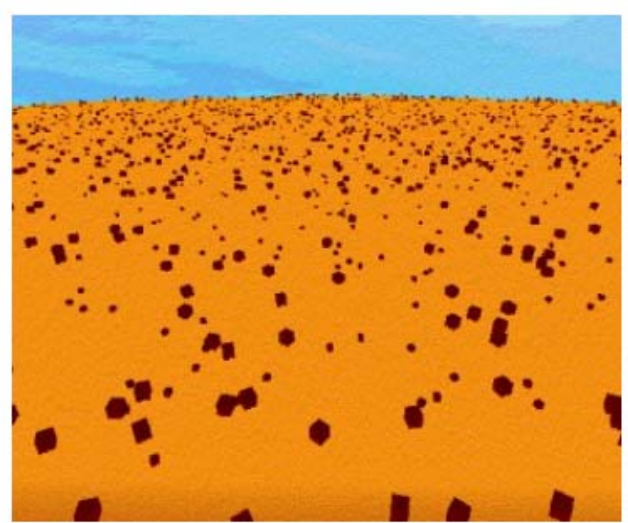

Figure 8. Virtual terrain of the Viking 1 landing site modeled within the Tumbleweed Simulator. 
would need to be stored at any given time. Another improvement area identified in this analysis was the modeling of surface winds within the Tumbleweed Simulator environment. Currently, there is limited data describing wind conditions on the surface of Mars. Detailed models exist for replicating the environment of the upper atmosphere, but in general there is little known about the wind profile and boundary layer effects on the Martian surface. In order to provide a realistic dynamic simulation, the Tumbleweed Simulator will need to address these environmental modeling weaknesses.

\section{Question 2: Can a realistic Tumbleweed rover design be modeled within the simulator?}

Two different Tumbleweed design configurations were modeled within the simulator, a $3 \mathrm{~m}$ and $5 \mathrm{~m}$ radius rover, both with a mass of $20 \mathrm{~kg}$. At this point in the study, a $20 \mathrm{~kg}$ mass for the Tumbleweed rover was deemed

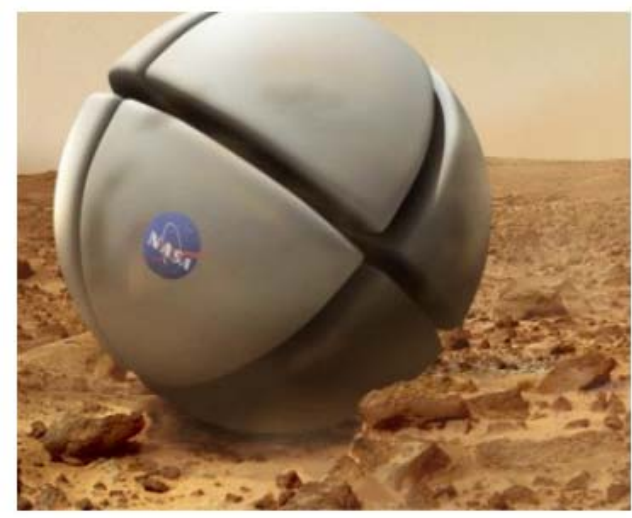

Figure 9. Artist's rendition of a Tumbleweed concept.

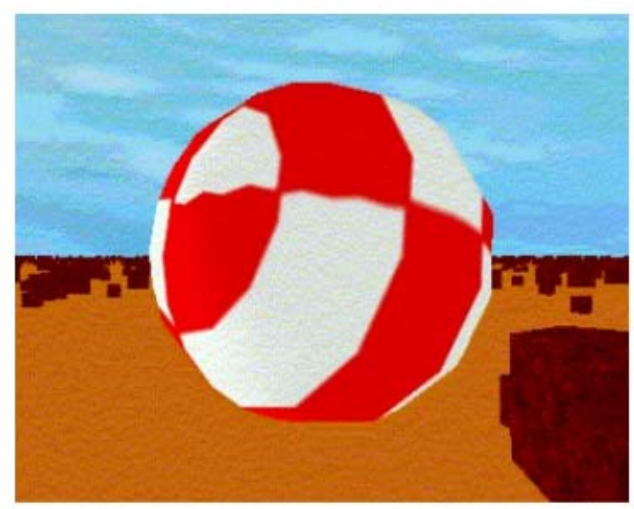

Figure 10. Virtual representation of the Tumbleweed concept from Fig. 9 created within the Tumbleweed Simulator. more realistic than the $10 \mathrm{~kg}$ mass assumed for the feasibility studies discussed earlier. Figures 9 and 10 show the comparison between an artist's rendition of one particular Tumbleweed design configuration and the virtual Tumbleweed modeled within the Tumbleweed Simulator.

The major obstacle to modeling a Tumbleweed rover within the simulator is correctly modeling a flexible structure or at least the dynamic losses that are associated with a flexible structure impacting and rolling on a non-rigid surface. The current version of the Tumbleweed Simulator models the Tumbleweed rover as a rigid body. All impacts are treated as rigid body, "billiard ball" collisions. The inherent flexibility the structure would actually have is accounted for somewhat by utilization of a coefficient of restitution. However, dynamic losses due to structural rolling resistance are not accounted for. In order to truly replicate the motion of a Tumbleweed rover, these dynamic losses will need to be accounted for in future analysis tools. High fidelity finite element tools exist that can accurately model impacts. These tools are, however, highly taxing on computational resources and are simply impractical for Monte Carlo type simulations. Alternative methods will need to be explored that can approximate the dynamic behavior of a Tumbleweed rover. One possibility might be to use a simplified modeling approach that incorporates simple physics relationships in parallel with empirical test data to apply correctional factors to the tool.

There are two other areas that the simulator should be improved regarding modeling of a Tumbleweed rover's design configuration. First, the coefficient of drag of the Tumbleweed is hard coded into the current version as that of a sphere at low Reynolds numbers $\left(C_{D}=0.5\right)$. Many of the current rover designs that have undergone wind tunnel testing at LaRC have much higher coefficients of drag $\left(C_{D}>1\right)^{4}$. Being able to vary the drag coefficient of the rover within the Tumbleweed simulator will be required to analyze these other configurations.

\section{Question 3: Can anything be learned about the dynamic behavior of the Tumbleweed rover from the tool in} its current state of development?

With the terrain and Tumbleweed rover modeled within the simulator to the best of its current ability, the next step in the evaluation was to set up and run a series of Monte Carlo simulations to take a preliminary look at the dynamic behavior of a Tumbleweed rover. The two rovers ( $3 \mathrm{~m}$ and $5 \mathrm{~m}$ radius) were run simultaneously over the simulated terrain with an initial separation of approximately $300 \mathrm{~m}$ to avoid Tumbleweed-to-Tumbleweed collisions. Their initial positions were slightly varied for each Monte Carlo iteration so that the path the rovers took across the terrain would be different for each run. The simulation consisted of 2000 iterations. 
Figure 11 shows the path taken across the simulated Viking 1 landing site terrain by the $5 \mathrm{~m}$ radius Tumbleweed rover over a 120 second time interval. The blown up area shows a segment of the Tumbleweed's path where there was an impact with a rock. The rover is thrown in the opposite direction as a result of the collision until eventually the $7 \mathrm{~m} / \mathrm{s}$ wind counteracts its backward motion and it resumes its movement in the down wind direction.

Figures 12 and 13 show the distribution of the 2000 Tumbleweed runs across the simulated terrain over a 120 second time interval for the $3 \mathrm{~m}$ and $5 \mathrm{~m}$ radius rovers, respectively. Each point represents the location of the rover on the terrain at the end of the 120 seconds for one of the 2000 Monte Carlo iterations. In the case of the $3 \mathrm{~m}$ radius Tumbleweed, the data is fairly uniformly scattered with the majority of the rovers being located $100 \mathrm{~m}$

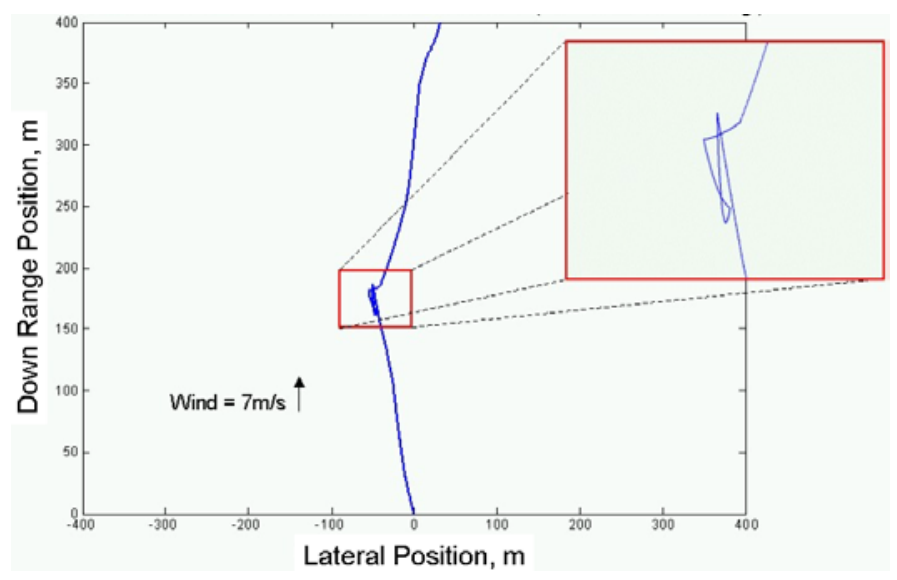

Figure 11. Simulated Tumbleweed rover path from one Monte Carlo iteration; $t=120 \mathrm{~s}, R_{t}=5 \mathrm{~m}, M_{t}=20 \mathrm{~kg}$.

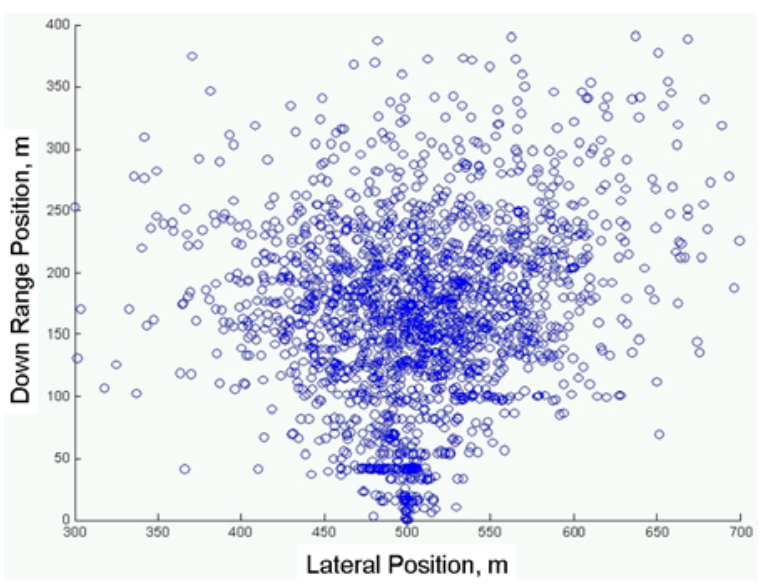

Figure 12. Tumbleweed rover distribution of 2000 Monte Carlo runs with the Tumbleweed Simulator; $R_{t}=3 \mathrm{~m}, M_{t}=20 \mathrm{~kg}$.

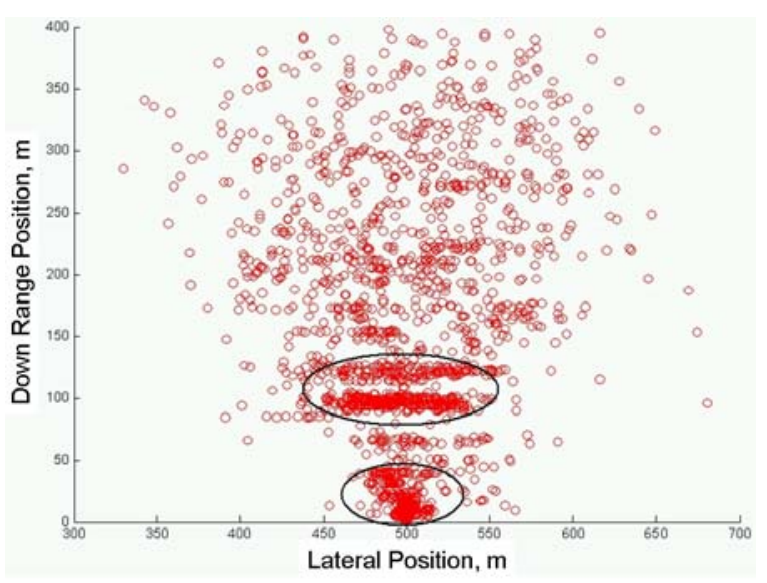

Figure 13. Tumbleweed rover distribution of 2000 Monte Carlo runs with the Tumbleweed Simulator; $R_{t}=5 \mathrm{~m}, M_{t}=20 \mathrm{~kg}$.

to $250 \mathrm{~m}$ downrange. For the $5 \mathrm{~m}$ radius Tumbleweed, one would expect the scatter to look similar to that of the $3 \mathrm{~m}$ Tumbleweed with the only major difference being that the larger diameter rovers would on average be able to move further down range in the same 120 seconds. Looking at Fig. 13, this is seen to some degree, but not as much as would be expected. Additionally, it can be seen that there are some strange concentrations of stuck Tumbleweeds (circled regions of the plot) in the first $150 \mathrm{~m}$ downrange for the larger $5 \mathrm{~m}$ radius rover.

Figures 14 and 15 display histograms of the distributions over the simulated terrain for the $3 \mathrm{~m}$ and $5 \mathrm{~m}$ radius rovers. The concentrations of the $5 \mathrm{~m}$ radius rovers discussed above become even more apparent when looking at the histograms. For the $3 \mathrm{~m}$ radius Tumbleweed, approximately 360 of the $2000(18 \%)$ iterations performed show the rover to be located 0 to $100 \mathrm{~m}$ downrange after 120 seconds. For the $5 \mathrm{~m}$ radius Tumbleweed, approximately 890 of the $2000(45 \%)$ iterations are located in the same downrange region over the same time interval.

One possible hypothesis for this discrepancy is directly related to the parameters selected for the simulated terrain within the Tumbleweed Simulator. As discussed earlier, the terrain defined was $1 \mathrm{~km}^{2}$ with a total of 10,000 randomly placed $1 \mathrm{~m}$ or $2 \mathrm{~m}$ cubic rocks. On average, this works out to be approximately 1 rock placed every $10 \mathrm{~m}$. Coincidentally, $10 \mathrm{~m}$ is also the diameter of the larger Tumbleweed rover. 


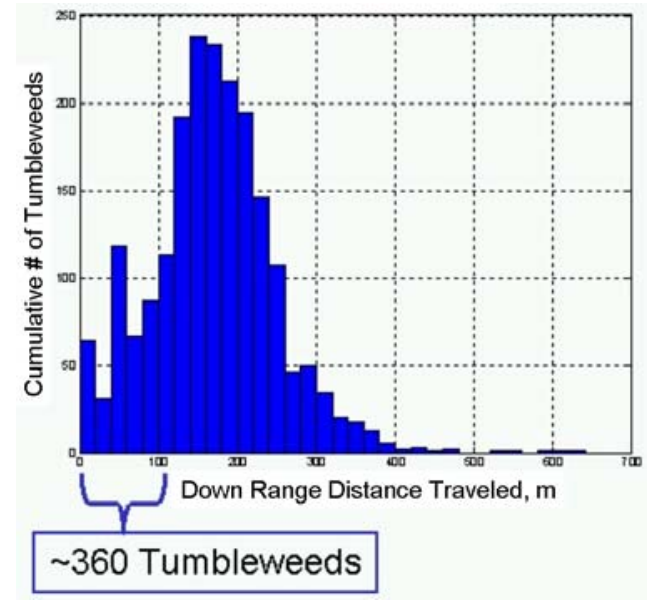

Figure 14. Histogram representation of Fig. 12; $R_{t}=3 \mathrm{~m}, M_{t}=20 \mathrm{~kg}$.

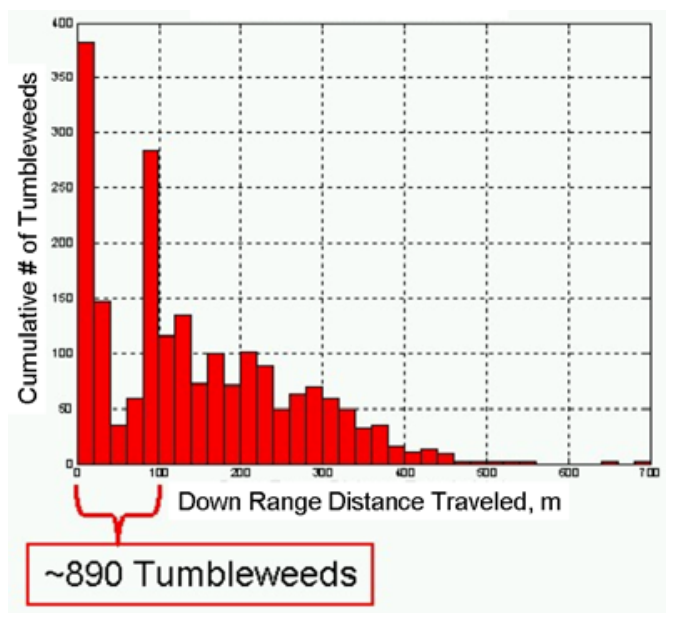

Figure 15. Histogram representation of Fig. 13; $R_{t}=5 \mathrm{~m}, M_{t}=20 \mathrm{~kg}$.

There are two basic ways in which a Tumbleweed rover can navigate past a rock; one is to go around it, and the other is to go over top of it. From a static analysis viewpoint, the $7 \mathrm{~m} / \mathrm{s}$ wind used in the simulation is not sufficient to push either of the Tumbleweed rovers from a rest over any of the $1 \mathrm{~m}$ or $2 \mathrm{~m}$ rocks. In other words, recalling Eq. (1) from section II, for $3 \mathrm{~m}$ and $5 \mathrm{~m}$ radius Tumbleweeds in a $7 \mathrm{~m} / \mathrm{s}$ wind, $M_{F w}$ will always be less than the sum of $M_{W}$ and $M_{N o}$. Since the Tumbleweeds start from rest at the beginning of each iteration and cannot reach a speed greater than the $7 \mathrm{~m} / \mathrm{s}$ wind acting on them, in order to navigate through the simulated terrain the rovers will have to go around the rocks. As can be seen in Fig. 16, the $3 \mathrm{~m}$ radius Tumbleweed has a distinct advantage due to its smaller size for navigating around and between the rocks of this particular terrain. From a dynamic standpoint, the initial velocity $\left(V_{i}\right)$ of the rover, its radius, and the size of the rock it is impacting will determine whether or not the collision will result in the rover bouncing up and over the rock or up and in the opposite direction from which it originally came from. More specifically, in order for the rover to bounce up and over the rock $\theta_{T}$, the post impact trajectory angle of the Tumbleweed (see Fig. 4), would have to be less than $90^{\circ}$, and the post impact bounce height would have to be higher than the height of the impacted rock.

Using the information and method presented in section II-B regarding collision analysis, the dynamic conditions required for going over the top of a given rock can be approximated. The calculations were carried out for the rock sizes and Tumbleweed rover configurations used in this evaluation and are summarized in Table 2. From these calculations, it can be seen that of the $3 \mathrm{~m}$ and $5 \mathrm{~m}$ radius Tumbleweeds, the $5 \mathrm{~m}$ rover is the only one of the two that can dynamically conquer the rocks on the simulated terrain, but only the $1 \mathrm{~m}$ rocks. Neither rover is

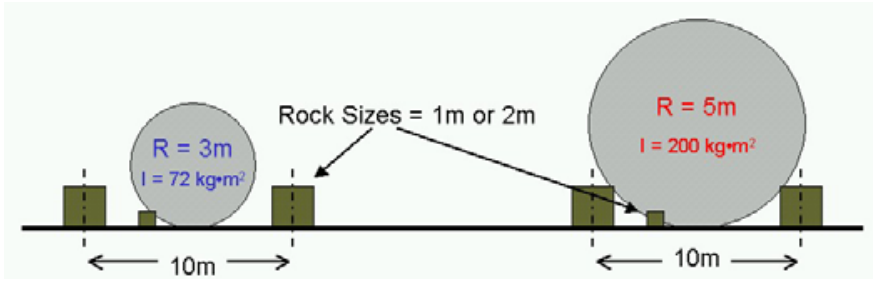

Figure 16. Illustration of how $3 \mathrm{~m}$ Tumbleweed can navigate the simulated terrain better than the $5 \mathrm{~m}$ Tumbleweed. Inertias assume a uniform density sphere. capable of dynamically conquering the $2 \mathrm{~m}$ rocks with the relatively mild $7 \mathrm{~m} / \mathrm{s}$ wind. The $5 \mathrm{~m}$ Tumbleweed would need an initial velocity of approximately $3 \mathrm{~m} / \mathrm{s}$ to bounce up high enough to go over top of the $1 \mathrm{~m}$ rock. For the other cases, $\theta_{T}$ is always greater than $90^{\circ}$. Consequently, it does not matter what initial velocity is obtained before the collision, the Tumbleweed rovers in these cases will always bounce back in the opposite direction from which they came.

Table 2. Requirements to dynamically overcome $1 \mathrm{~m}$ and $2 \mathrm{~m}$ rocks.

\begin{tabular}{|c|c|c|c|c|}
\hline & \multicolumn{2}{|c|}{ 3 m Radius Tumbleweed } & \multicolumn{2}{c|}{$\mathbf{5}$ m Radius Tumbleweed } \\
\hline Rock Size (m) & $\theta_{T}\left({ }^{\circ}\right)$ & $V_{i}(\mathrm{~m} / \mathrm{s})$ & $\theta_{T}\left({ }^{\circ}\right)$ & $V_{i}(\mathrm{~m} / \mathrm{s})$ \\
\hline $\mathbf{1} \times 1 \times 1$ & 93 & NA & 71 & $\sim 3$ \\
\hline $\mathbf{2} \times \mathbf{2} \times \mathbf{2}$ & 140 & NA & 103 & NA \\
\hline
\end{tabular}


From the preliminary analysis discussed in this section, a hypothesis can be generated that would suggest that because of the properties of this particular terrain and the chosen design configuration of the Tumbleweeds, the $3 \mathrm{~m}$ radius rovers are more efficient at navigating through this particular rock field than the $5 \mathrm{~m}$ radius rovers. In the first $100 \mathrm{~m}$ to $150 \mathrm{~m}$ down range, the $5 \mathrm{~m}$ radius Tumbleweeds do not have sufficient time to accelerate to the $3 \mathrm{~m} / \mathrm{s}$ necessary to bounce up and over the $1 \mathrm{~m}$ rocks. As a result, they will bounce backward and eventually become lodged, as shown in Fig. 16, much more frequently than the $3 \mathrm{~m}$ radius rovers. The few $5 \mathrm{~m}$ radius Tumbleweeds that do make it past the first $150 \mathrm{~m}$ perform as expected and generally travel further down range than the $3 \mathrm{~m}$ radius Tumbleweeds. Furthermore, even though the $3 \mathrm{~m}$ radius rovers cannot ever bounce up and over any of the rocks in this terrain (because the relationship between the dimensions of the rocks and the rover will never allow $\theta_{T}$ to be greater than $90^{\circ}$ ), they will always have an easier time going around the rocks laterally simply because of their smaller diameter.

\section{Conclusion}

The purpose of this paper is to discuss the results of an initial feasibility studies and a preliminary dynamics analysis of the Mars Tumbleweed rover concept. The results of the feasibility studies have served to aide the LaRC Tumbleweed team in visualizing the dynamic behavior of a Tumbleweed rover. The evaluation and development of the Tumbleweed Simulator has served to guide future dynamics analysis efforts. Additionally, even though the Tumbleweed Simulator is very much still a developmental and low fidelity analysis application, it has nonetheless turned out to be a valuable visualization tool at the very least. Finally, it is important to reiterate that this is a preliminary analysis and effects of rock sizes on Tumbleweed navigability will obviously need to be looked at in more detail. However, the analysis presented in this paper has yielded an interesting result that might not have been noticed unless a Monte Carlo type simulation was studied. Consequently, a preliminary conclusion can be drawn about the relationship between Tumbleweed size and terrain navigability that at the very least challenges the intuitive assumption that bigger Tumbleweeds with larger surface areas are always more proficient at achieving maximum down range distance. In fact, this analysis shows that it may actually turn out that the optimal Tumbleweed diameter is highly dependent on the properties of the terrain it is intended to traverse.

\section{References}

${ }^{1}$ Antol, J. A., Calhoun, P. C., Flick J. J., Hajos G. J., Kolacinski R., Minton D., Owens R., and Parker J. R., "Low Cost Mars Surface Exploration: The Mars Tumbleweed," NASA/TM-2002-212411.

${ }^{2}$ Horne, W. B., Trafford, W. L., "Influence of Tire Tread Pattern and Runway Surface Condition on Braking Friction and Rolling Resistance of a Modern Aircraft Tire,” NASA TN-D-1376, 1962.

${ }^{3}$ Calhoun, P. C., Harris, S. B., Raiszadeh, B., Zaleski K. D., "Conceptual Design, Dynamics Modeling, and Videogrammetric Testing of a Novel Tumbleweed Rover", AIAA-2005-0247, 43rd AIAA Aerospace Sciences Meeting and Exhibit, Reno, NV, January 10-13, 2005.

${ }^{4}$ Strickland C., Keyes J., "Wind Tunnel Tests to Determine Drag Coefficients for the Mars Tumbleweed," AIAA-2005-0248, 43rd AIAA Aerospace Sciences Meeting and Exhibit, Reno, NV, January 10-13, 2005.

${ }^{5}$ Golombek, M., Rapp, D., "Size-Frequency Distributions of Rocks on Mars and Earth Analog Sites: Implications for Future Landed Missions," Journal of Geophysical Research, Vol. 102, No. E2, 27 Feb. 1997, pp. 4117, 4129. 